\title{
Plasma and Cerebrospinal Fluid Inflammation and the Blood Brain Barrier in Older Surgical Patients: The Role of Inflammation after Surgery for Elders Study
}

Sarinnapha Vasunilashorn ( $\nabla$ svasunil@bidmc.harvard.edu )

Beth Israel Deaconess Medical Center

Long $\mathrm{H}$. Ngo

Beth Israel Deaconess Medical Center

Simon T. Dillon

Beth Israel Deaconess Medical Center

Tamara G Fong

Beth Israel Deaconess Medical Center

Becky C Carlyle

Massachusetts General Hospital

Pia Kivisakk

Massachusetts General Hospital

Bianca A Trombetta

Massachusetts General Hospital

Kamen V Vlassakov

Brigham and Women's Hospital

Lisa J Kunze

Beth Israel Deaconess Medical Center

Steven E Arnold

Massachusetts General Hospital

Zhongcong Xie

Massachusetts General Hospital

Sharon K Inouye

Hebrew SeniorLife

Towia A Libermann

Beth Israel Deaconess Medical Center

Edward R Marcantonio

Beth Israel Deaconess Medical Center 
Research

Keywords: inflammation, neuroinflammation, plasma, cerebrospinal fluid, blood-brain barrier

Posted Date: December 1st, 2020

DOI: https://doi.org/10.21203/rs.3.rs-115991/v1

License: (c) (i) This work is licensed under a Creative Commons Attribution 4.0 International License. Read Full License 


\section{Abstract \\ Background}

Our understanding of the relationship between plasma and cerebrospinal fluid (CSF) remains limited, which poses an obstacle to the identification of blood-based markers of neuroinflammatory disorders. To better understand the relationship between peripheral and central nervous system (CNS) markers of inflammation before and after surgery, we aimed to: examine whether surgery compromises the bloodbrain barrier (BBB), evaluate postoperative changes in inflammatory markers, and assess the correlations between plasma and CSF levels of inflammation.

\section{Methods}

We examined the Role of Inflammation after Surgery for Elders (RISE) study of adults aged $\geq 65$ who underwent elective hip or knee surgery under spinal anesthesia who had plasma and CSF samples collected at baseline and postoperative 1 month (PO1MO) $(n=29)$. Plasma and CSF levels of three inflammatory markers previously identified as increasing after surgery were measured using enzymelinked immunosorbent assay: interleukin-6 (IL-6), C-reactive protein (CRP), and chitinase 3-like protein (also known as YKL-40). Integrity of the BBB was computed as the ratio of CSF/plasma albumin levels (Qalb). Mean Qalb and levels of inflammation were compared between baseline and P01MO. Spearman correlation coefficients were used to determine correlation between biofluids. For the plasma-CSF biofluids with significant correlations, we determined whether the markers were associated by using linear regression models.

\section{Results}

Mean Qalb did not change between baseline and P01MO. Plasma and CSF levels of IL-6, CRP, and YKL40 were higher on $\mathrm{P01 \textrm {MO }}$ relative to baseline, with a disproportionally higher increase in CSF levels relative to plasma levels (IL-6 doubled and CRP tripled in CSF). Significant plasma-CSF correlations for CRP (baseline $r=0.70$ and P01MO $r=0.89, p<.01$ for both) and IL-6 (P01MO $r=0.48, p<.01$ ) were observed, with higher correlations on P01M0 compared with baseline.

\section{Conclusions}

In this elective surgical sample of older adults, BBB integrity was similar between baseline and P01MO, inflammation levels were higher P01MO than baseline, and plasma-CSF correlations were observed for CRP and IL-6. Our identification of potential promising plasma markers of inflammation in the CNS may facilitate the early identification of patients at greatest risk for neuroinflammation and its associated adverse cognitive outcomes. 


\section{Background}

Insights into the relationship of plasma and cerebrospinal fluid (CSF) biomarkers may help to advance our pathophysiologic understanding of how peripheral events like surgery might trigger neuroinflammation. Our understanding of the inter-relationship between plasma and CSF remains incomplete, and as a consequence, our ability to identify blood-based biomarkers of the surgical effect, including inflammation and neuroinflammation, remains limited. Recent data support an emerging model highlighting the potential associated downstream consequences of surgery: individuals predisposed to a heightened inflammatory response when exposed to an acute stressor, such as surgery or infection, are at increased risk for longer-term adverse outcomes (Marcantonio, 2012). Under certain conditions, these systemic inflammatory mediators are hypothesized to activate brain microglia, leading to neuroinflammation, which if sustained, may cause permanent neuronal injury (as illustrated in Marcantonio, 2012).

From this posited neuroinflammatory hypothesis, two prominent questions remain. First, how does an acute stressor such as surgery impact the BBB after a prolonged period of time? Second, can we identify blood-based markers indicative of neuroinflammation? Such questions require understanding the complex nature of molecular dynamics underscoring the protein levels in peripheral blood and the central nervous system (CNS), which can be influenced by many factors.

To better understand the complex relationship between peripheral and CNS markers of inflammation before and after surgery, we evaluated three key knowledge gaps in a study of older adults undergoing major elective surgery under spinal anesthesia. Our specific aims were: (1) to examine whether surgery compromises the blood-brain barrier (BBB, measured from CSF-plasma albumin ratio [Qalb]) one month post-surgery; (2) to evaluate whether changes in levels of inflammatory markers following surgery are greater in plasma and CSF at one month post-operation (P01MO) relative to preoperation (PREOP or baseline); and (3) to examine 3 previously identified plasma markers of inflammation associated with surgery for their correlations with CSF levels: interleukin (IL)-6, C reactive protein (CRP) and chitinase-3 Like Protein 1 (CHI3L1, also known as YKL-40). We hypothesized that: 1) surgery would compromise the $\mathrm{BBB}$, resulting in higher permeability of proteins observed one month following surgery; 2a) mean P01MO levels of all three inflammatory markers would be higher on P01MO compared to PREOP; 2b) CSF levels would exhibit disproportionately higher increases in these inflammatory markers following surgery compared with plasma; 3a) plasma markers of inflammation would have high correlations with CSF, and 3b) higher plasma-CSF correlations would be observed on P01MO compared with PREOP.

\section{Methods}

\section{Study Sample}

The Role of Inflammation after Surgery in Elders (RISE) study is a cohort study aimed to assess the correlation of blood plasma, CSF, and imaging biomarkers of inflammation in patients aged 65 year or 
older who underwent elective hip or knee arthroplasty under spinal anesthesia. The overall study design and protocol have been described (Hshieh et al., 2019). Briefly, patients were enrolled if they had planned admission for at least 24 hours and surgery scheduled at least 15 days in advance to allow time for preoperative testing. Exclusion criteria have been previously described, and include safety exclusions for lumbar puncture and magnetic resonance imaging.

The Institutional Review Board of Partners Healthcare System (Massachusetts General Hospital, Brigham and Women's Hospital, Brigham and Women's Faulkner Hospital) approved all study procedures, with ceded review from Beth Israel Deaconess Medical Center and Hebrew SeniorLife, the study coordinating center.

\section{Specimen Collection}

Phlebotomy was performed on patients at three time points: baseline (at home or during preadmission testing clinic visit), postoperative day 1 (POD1), and approximately one month postoperatively (P01M0). During processing, plasma and cellular material were separated using low-speed centrifugation (1500 relative centrifugal force [rcf]), subaliquoted, and stored at $-80 \mathrm{C}$.

CSF was acquired preoperatively during induction of spinal anesthesia (baseline) and at P01MO via lumbar puncture. CSF was collected via dropwise collection or aspiration directly into collection tubes. To minimize potential blood contamination of the CSF, samples were centrifuged at $1000 \mathrm{rcf}$ for 10 minutes prior to storage at $-80^{\circ} \mathrm{C}$ in low absorption polypropylene tubes. This paper focuses on the baseline and P01MO time points since CSF was only available at these two time points.

\section{Immunoassays}

Plasma and CSF levels of three inflammatory markers (IL-6, CRP, and YKL-40) and of albumin were measured using sandwich assays: the fully automated Ella System (ProteinSimple San Jose, CA) for the inflammatory markers, and an enzyme-linked immunosorbent assay from Abcam (Cambridge, MA; ab108788) run on a semiautomatic Tecan Freedom Evo liquid handling platform (Männedorf, Switzerland) for albumin. Qalb was defined as CSF albumin/plasma albumin $\times 10^{-3}$. Coefficient of variations (CVs) of duplicate measures were generally $<5 \%$. If a CV was $>10 \%$, the assay was repeated.

\section{Statistical Analysis}

To determine correlation within biofluids (i.e., plasma-plasma or CSF-CSF) and between biofluids (i.e., plasma-CSF), we examined Spearman correlation coefficients. All analyses were conducted using SAS 9.4 (SAS Institute, Cary, NC).

\section{Results}

Table 1 reports the characteristics of our study sample, presenting means and standard deviations, as well as proportions, for the sample of patients with complete plasma and CSF biospecimen data at both 
baseline and P01MO ( $\mathrm{n}=29)$. Patients were on average age 75 and mostly female, $10 \%$ had $\geq 2$ Charlson comorbidities, and all patients underwent spinal anesthesia alone. Fifty-two percent of patients underwent total knee arthroplasty and $48 \%$ underwent total hip arthroplasty. The average hospital length of stay was 3.2 days.

Table 2 reports the distributions of albumin, IL-6, CRP, and YKL-40 in the sample with complete plasma and CSF biospecimen data $(n=29)$. Between baseline and P01MO, we observed three general patterns. First, mean Qalb was similar between the baseline and P01MO time points ( 6.42 for both); a small number of patients had Qalb $\geq 9.0$ (4 patients [14\%] at baseline and 2 patients $(7 \%)$ on PO1MO, including the one patient having Qalb $\geq 9.0$ at both time points), indicating compromised BBB (Bowman et al., 2007). Second, IL-6 and CRP levels increased for both plasma and CSF. Average plasma IL-6 levels increased from $3.7 \mathrm{pg} / \mathrm{ml}$ (baseline) to $5.4 \mathrm{pg} / \mathrm{ml}$ (P01MO), and average CSF IL-6 doubled from 4.0 $\mathrm{pg} / \mathrm{ml}$ (baseline) to $8.3 \mathrm{pg} / \mathrm{ml}$ (PO1MO). Similarly, increases in CRP levels were observed between the two time points: average plasma CRP levels increased from $7.4 \mathrm{mg} / \mathrm{l}$ (baseline) to $8.2 \mathrm{mg} / \mathrm{l}$ (PO1MO), and average CSF CRP tripled between baseline $(0.01 \mathrm{mg} / \mathrm{l})$ and P01MO $(0.03 \mathrm{mg} / \mathrm{l})$. Third, YKL-40 increased in plasma only $(96.8 \mathrm{ng} / \mathrm{ml}$ [baseline] to $135.7 \mathrm{ng} / \mathrm{ml}$ [P01MO]), while CSF levels remained near equivalent at baseline $(280.1 \mathrm{ng} / \mathrm{ml})$ and PO1MO $(280.0 \mathrm{ng} / \mathrm{ml})$. When we conducted the same analyses using the full dataset of RISE study patients with blood and CSF at PREOP $(n=57)$ and at P01MO $(n=42)$ or without blood and/or CSF at either time point, the overall findings remained similar (results available upon request).

Table 3 reports the Spearman correlation coefficients of plasma and CSF inflammatory markers at baseline ( $n=29)$. Between biofluids (plasma and CSF; shown in red), three significant correlations were identified - all including CSF levels of CRP with plasma levels at baseline of: itself (CRP: $r=0.70, p<.01$ ), IL-6 $(r=0.58, p<.01)$, and YKL-40 $(r=0.43, p<.05)$.

Table 4 lists the Spearman correlation coefficients of plasma and CSF inflammatory markers at P01MO $(n=29)$. Similar to baseline, IL-6, YKL-40, and CRP plasma levels were significantly correlated with CRP CSF levels, all assessed at 1 month ( $r=0.47,0.59$, and 0.89 , respectively). Additionally, IL- 6 plasma and IL6 CSF levels were moderately correlated at P01MO $(r=0.48, p<.01)$.

We additionally examined the Spearman correlation coefficients of plasma and CSF inflammatory markers between baseline and P01MO. Within both plasma and CSF, every protein was significantly correlated with itself between the two time points. Spearman r's for plasma IL-6, CRP, and YKL-40 measured on baseline and P01MO were: $0.49,0.52$, and 0.80 ( $p<.01$ for all); Spearman r's for CSF IL-6, CRP, and YKL-40 measured on baseline and P01MO were: $0.50,0.58$, and 0.93 ( $p<.01$ for all). The only significant correlation between biofluids at the two time points was observed between baseline levels of YKL-40 plasma and P01MO CSF levels of CRP $(r=0.51, p<.01)$.

Baseline Qalb (our measure of BBB integrity) was correlated with inflammatory markers at both baseline and P01MO. Qalb at baseline was correlated with: (1) baseline CRP CSF levels ( $r=0.48, p<.01),(2)$ P01MO 
levels of plasma CRP, CSF CRP, and CSF IL-6 ( $r=0.45,0.47$, and 0.38 [respectively], $p<.05$ for all). There was good correlation between Qalb baseline and Qalb P01MO $(r=0.64, p<.01)$.

\section{Discussion}

In this study of older adults undergoing major noncardiac surgery, we found evidence in support of two of our three hypotheses (summarized in Fig. 1). Our first hypothesis was not supported; we need did not observe compromises in the integrity of the BBB between PREOP and P01MO based on the CSF-albumin ratio (Hypothesis 1). For our second hypothesis, we found that both plasma and CSF mean levels of IL-6, CRP, and YKL-40 were higher on P01MO relative to PREOP levels (Hypothesis 2a). Additionally, there was a disproportionately higher increase in CSF levels relative to plasma levels between PREOP and P01MO for: IL-6 (doubled in CSF) and CRP (tripled in CSF) (Hypothesis 2b). For our third hypothesis, we observed significant plasma-CSF correlations for CRP (PREOP and P01MO) and IL-6 (P01MO only) (Hypothesis 3a), with higher correlations on P01MO compared to PREOP (Hypothesis $3 b$ ).

At both baseline and P01MO, we found that plasma and CSF levels of CRP were well correlated, and that levels of plasma IL- 6 and levels of plasma YKL-40 were correlated with CSF CRP. At P01MO only, plasma and CSF levels of IL- 6 were correlated. Despite these between biofluid correlations, we were ultimately unable to identify promising blood-based inflammatory markers of CNS inflammation, based on our knowledge of the origins of these inflammatory markers. As an example, since CRP is felt to be solely produced in the periphery (predominantly in the liver), it is more probable that our findings reflect a 'leaking' of CRP from the periphery (i.e., plasma) into the CNS (i.e., CSF) than 'leakage' from the CNS into the periphery. Interestingly, the increased levels of CSF inflammatory proteins at P01MO occurred despite no change in Qalb, suggesting that BBB integrity for other proteins may not be fully reflected in this measure. Given this, it is unlikely that plasma CRP, IL-6, or YKL-40 are promising blood-based markers of CSF inflammation. We also found that: (1) peripheral markers of inflammation correlated well with other peripheral inflammatory markers within the same time point, and (2) for plasma and CSF, each inflammatory marker was correlated with itself between the baseline and P01MO time points.

Although not observed in our study, compromises in BBB have been associated with surgery in humans and animal models. In mice, anesthesia and/or surgery may induce age-associated BBB permeability, as determined by immunohistochemistry imaging and spectrophotometric quantification (Yung et al., 2017). Among patients undergoing cardiac surgery, postsurgical disruption of the BBB detected using magnetic resonance imaging (MRI) was observed in $47 \%$ of the 19 patients, all of whom had no clinical evidence of a stroke or delirium at the time of gadolinium administration or the MRI scan (Merino et al., 2013). The absence of changes in BBB permeability between baseline and P01MO in our healthy sample of older RISE patients may be due to the fact that post-surgery disruptions in the BBB may have resolved by the 1 month post-surgery time point or may be related to the poor sensitivity of Qalb to detect changes in BBB permeability. Ideally, we would have examined an alternate measure of BBB integrity or Qalb closer to the surgical event (e.g., postoperative day 1 [POD1]); however, we were limited in the measures we could assay and were unable to obtain CSF at the POD1 time point. 
Plasma and CSF correlations of inflammatory markers seem to be dependent on the characteristics of the study sample. Among 141 patients with Alzheimer's disease (AD), a good correlation between plasma and CSF levels of IL-6 levels was reported $(r=0.76, p<.001)$ (Sun et al., 2003). In contrast, no correlation between IL-6 plasma and CSF levels was observed in 173 older adults who were asymptomatic for AD ( $r$ $=0.16, p=.05$ ) (Bettcher et al., 2018). Our findings in older surgical adults (none of whom had known preexisting dementia, yielded a plasma-CSF correlation of IL-6 levels that was between the Sun et al. (2003) and Bettcher et al. (2018) publications $(r=0.48, p<.01$ on P01MO). This suggests that, for IL-6, the relationship between plasma and CSF may be influenced by factors associated with cognition (e.g., BBB integrity or presence of $A D$ ), although we were unable to adequately probe this possibility within our small sample. Although Qalb did not increase between baseline and P01MO in our sample, the plasma-CSF IL-6 correlation and association findings suggest the possibility of: (1) increased permeability for small proteins, such as IL- 6 (approximately $20 \mathrm{kDa}$ in size versus, for example, the size of albumin [about 60 $\mathrm{kDa}]$ ), (2) IL-6 may be stimulating CNS IL-6 production indirectly (irrespective of the integrity of the BBB), or (3) stimuli that induce IL-6 expression in peripheral blood mononuclear cells also induces the expression in microglial cells since plasma and CSF levels of IL-6 were observed to be relatively similar at baseline.

Among the inflammatory markers examined, we found preliminary evidence for the possibility of stronger plasma-CSF correlations at the P01MO time point. For instance, CRP exhibited medium to high correlations at baseline $(r=0.70, p<.01)$ and extremely high correlation at P01MO $(r=0.89, p<.01)$. These promising findings highlight the importance of further examining these relationships in larger surgical cohorts.

We highlight several study strengths. RISE applied state-of-the art approaches to the collection of biospecimens and detailed clinical data on older patients undergoing major surgery, including collection of plasma and CSF. Our empirically driven analysis examined potential correlations between plasma and CSF, with a focus on the longer-term effects of surgery (one month post-operation). This facilitated further probing of correlations and the integrity of the BBB following surgery over a longer time frame than may not have been previously observed in the literature.

Some study limitations warrant mention. First, this is a relatively small study which will need confirmation; yet it provides important descriptive information to better understand BBB in the context of the longer-term effects of surgery and to identify peripheral markers of neuroinflammation. Given the sample size, we did not adjust for multiple testing, control for covariates, or examine alternate means of considering nonlinearities in the relationship between plasma and CSF levels in our regression models. We ultimately employed a biomarker "correlational" discovery approach to generate hypotheses of peripheral-CNS relationships that will require confirmation in larger cohorts, for which we are currently enrolling and intend to pursue more rigorous statistical analyses in future work. Second, although we did not observe a notably strong correlation between plasma and CSF inflammatory markers in this small, selected sample, we acknowledge that an absence of a correlation does not indicate an inherent lack of peripheral markers of neuroinflammation. It may be that our limited set of inflammatory markers does not 
appropriately represent indicators of inflammation in the CNS. For instance, macrophage inflammatory protein (MIP-1 $\beta$, also known as CCL4), previously observed to be moderately correlated in plasma and CSF among older adults without AD ( $r=0.55$, Bettcher et al., 2018), may be a promising additional inflammatory marker for future examination. Ultimately, the identification of a blood-based marker of neuroinflammation will require identification of an inflammatory marker that is produced entirely, or nearly entirely in the CNS (and not in the periphery). Third, at baseline, there was more variability in the time interval between blood and plasma acquisition compared to the collection of both biofluids at P01M0, which was almost always done at the same time. As previously noted, this may explain the generally stronger correlations observed on P01MO relative to baseline. Fourth, since all patients underwent spinal anesthesia, we cannot rule-out possible protective effects where spinal anesthesia may mediate the relationships observed. Last, we use CSF levels of inflammation as the "gold standard" for neuroinflammation given the absence of an alternative approach, such as brain imaging markers or brain tissue in the RISE study.

In summary, we found that all three inflammatory markers were higher at 1 month post-surgery than at baseline, with a higher increase in CSF levels relative to plasma levels between the two time points. In contrast, the integrity of the BBB was similar between the two time points. The plasma-CSF correlation results suggest that CRP plasma-CSF correlations are high at all time points, likely due to leakage of peripheral CRP into the CNS. Alternatively, IL- 6 measured on P01MO may be a promising blood-based marker of neuroinflammation. Future studies in larger surgical populations that further probe the possibility of IL- 6 and other potential blood-based markers of inflammation in the CNS will facilitate the identification of blood-based markers of neuroinflammation and understanding of CNS inflammatory disorders. Our ability to identify such a marker would facilitate the early detection of patients with greatest risk for neuroinflammation and its associated adverse cognitive outcomes.

\section{Abbreviations}

BIDMC, Beth Israel Deaconess Medical Center; BWH, Brigham and Women's Hospital; HMS, Harvard Medical School; HSL, Hebrew SeniorLife; MGH, Massachusetts General Hospital; PI, principal investigator; UNL, University of Nebraska-Lincoln.

\section{Declarations}

\section{Ethics approval and consent to participate}

The Institutional Review Board of Partners Healthcare System (Massachusetts General Hospital, Brigham and Women's Hospital, Brigham and Women's Faulkner Hospital) approved all study procedures, with ceded review from Beth Israel Deaconess Medical Center and Hebrew SeniorLife, the study coordinating center.

\section{Consent for publication}


Not applicable.

\section{Availability of data and materials}

The datasets analyses during the current study are available from the corresponding author on reasonable request.

\section{Competing interests}

The authors have no competing financial interests to disclose.

\section{Funding}

This research was supported by the Alzheimer's Drug Discovery Foundation [SKI], and the National Institute on Aging grants (P01AG031720 [SKI], K01AG057836 [SMV], R03AG061582 [SMV], R24AG054529 [SKI], R01AG041274 [ZX], R21AG048600 [ZX], R01AG051658 [ERM, TAL], and K24AG035075 [ERM]), and the Alzheimer's Association (AARF-18-560786 [SMV]). Dr. Inouye holds the Milton and Shirley F. Levy Family Chair.

\section{Authors' contributions}

SMV, LHN, STD, TGF, SCC, PK, SEA, SKI, TAL, ERM conceived and designed the study. SMV, LHN, STD, TGF, SCC, PK BAT, KVK, LJK, SEA, ZX, SKI, TAL, ERM contributed to the acquisition and analysis of data. SMV drafted the manuscript. SMV LHN, STD, TGF, SCC, PK BAT, KVK, LJK, SEA, ZX, SKI, TAL, ERM contributed to the data interpretation, revision of the text and preparation of the tables and figures. All authors read and approved the final manuscript.

\section{Acknowledgements}

The authors gratefully acknowledge the contributions of the patients, family members, nurses, physicians, staff members, and members of the Executive Committee who participated in the Role of Inflammation after Surgery for Elders (RISE) Study.

\section{References}

1. Marcantonio ER. Postoperative delirium: a 76-year-old woman with delirium following surgery. JAMA. 2012;308:73-81.

2. Hshieh TT, Vasunilashorn SM, D’Aquila ML, Arnold SE, Dickerson BC, Fong TG, et al. The Role of Inflammation after Surgery for Elders (RISE) Study design, procedures, and cohort profile. Alzheimers Dementia (DADM) 2019;11:752-762. AM J Neuroradiol 2013;34:518-523.

3. Bowman GL, Kaye JA, Moore M, Waichunas D, Carlson NE, Quinn JF. Blood-brain barrier impairment in Alzheimer disease: Stability and functional significance. Neurology. 2007;68:1809-14. 
4. Yang S, Gu C, Mandeville ET, Dong Y, Esposito E, Zhang Y, Yang G, Shen Y, Fu X, Lo EH, Xie Z. Anesthesia and surgery impair blood-brain barrier and cognitive function in mice. Front Immunol. 2017;8:902.

5. Merino JG, Latour LL, Tso A, Lee KY, Kang DW, Davis LA, Lazar RM, Horvath KA, Corso PJ, Warach S. Blood-brain barrier disruption after cardiac surgery.

6. Sun YX, Minthon L, Wallmark A, Warkentin S, Blennow K, Janciauskiene S, et al. Inflammatory markers in matched plasma and cerebrospinal fluid from patients with Alzheimer's disease. Dement Geriatr Cogn Dis. 2003;16:136-44.

7. Bettcher BM, Johnson SC, Fitch R, Casaletto KB, Herrernan KS, Asthana S, Zetterberg H, Blennow K, Carlsson CM, Neuhaus J, Bendlin BB, Kramer JH. CSF and plasma levels of inflammation differentially relate to CNS markers of Alzheimer's disease pathology and neuronal damage. $\mathrm{J}$ Alzheimers Dis. 2018;62:385-97.

\section{Tables}

Table 1. Sample characteristics of the RISE study patients with complete biospecimen data (plasma and cerebrospinal fluid) at both baseline and postoperative 1 month

\begin{tabular}{|ll|}
\hline & Complete Biospecimen Data for BL and P01MO (N=29) \\
\hline Age, $\mathrm{M} \pm \mathrm{SD}$ & $74.8 \pm 4.6$ \\
\hline Female, $\mathrm{n}(\%)$ & $20(69 \%)$ \\
\hline Non-white, $\mathrm{n}(\%)$ & $2(7 \%)$ \\
\hline Charlson comorbidity index, $\mathrm{n}(\%)$ & \\
\hline 0 & $24(83 \%)$ \\
\hline 1 & $2(7 \%)$ \\
\hline $2+$ & $3(10 \%)$ \\
\hline Anesthesia type & \\
\hline Spinal alone, $\mathrm{n}(\%)$ & $29(100 \%)$ \\
\hline Surgery type, $\mathrm{n}(\%)$ & $15(52 \%)$ \\
\hline Total knee arthroplasty & $14(48 \%)$ \\
\hline Total hip arthroplasty & $3.2 \pm 0.8$ \\
\hline Hospital length of stay, M \pm SD & \\
\hline
\end{tabular}

Abbreviations: $\mathrm{BL}$ = baseline, $\mathrm{CAM}-\mathrm{S} \mathrm{LF}=$ Confusion Assessment Method-Severity long form, GCP = general cognitive performance, $\mathrm{M}=$ mean, $\mathrm{PO} 1 \mathrm{MO}$ = postoperative 1 month, $\mathrm{SD}=$ standard deviation 
Table 2. Distribution of markers within the analytic sample (data available at both PREOP and PO1MO, $\mathrm{N}=29$ )

\begin{tabular}{|c|c|c|c|c|c|}
\hline Time period & Biofluid & Mean \pm SD & Median & Min & Max \\
\hline \multicolumn{6}{|c|}{ 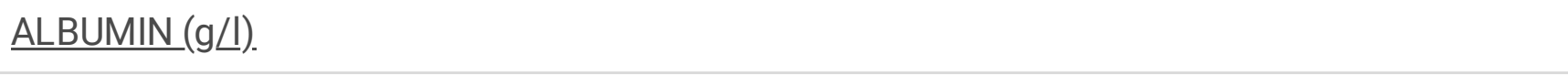 } \\
\hline \multirow[t]{3}{*}{ Baseline } & CSF & $0.18 \pm 0.07$ & 0.17 & 0.08 & 0.34 \\
\hline & Plasma & $28.64 \pm 7.33$ & 27.10 & 16.52 & 52.84 \\
\hline & CSF/plasma ratio $\left(\times 10^{-3}\right)$ & $6.42 \pm 2.62$ & 6.64 & 2.31 & 14.20 \\
\hline \multirow[t]{3}{*}{ P01M0 } & CSF & $0.17 \pm 0.06$ & 0.17 & 0.07 & 0.34 \\
\hline & Plasma & $28.06 \pm 7.48$ & 27.25 & 18.97 & 57.11 \\
\hline & CSF/plasma ratio $\left(\times 10^{-3}\right)$ & $6.42 \pm 2.76$ & 6.17 & 1.98 & 15.22 \\
\hline \multicolumn{6}{|c|}{ 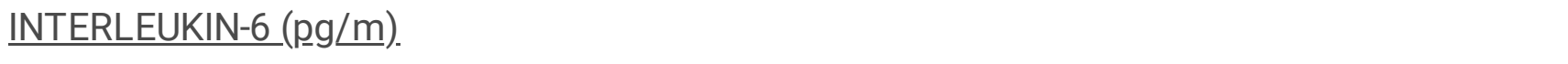 } \\
\hline \multirow[t]{2}{*}{ Baseline } & Plasma & $3.67 \pm 1.68$ & 3.21 & 1.52 & 7.33 \\
\hline & CSF & $4.05 \pm 1.58$ & 3.49 & 1.3 & 8.67 \\
\hline \multirow[t]{2}{*}{ P01M0 } & Plasma & $5.41 \pm 3.37$ & 4.37 & 2.23 & 15.90 \\
\hline & CSF & $8.25 \pm 23.46$ & 3.67 & 1.34 & 130.00 \\
\hline \multicolumn{6}{|c|}{ 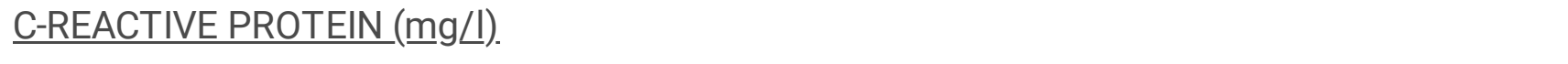 } \\
\hline \multirow[t]{2}{*}{ Baseline } & Plasma & $7.41 \pm 13.21$ & 2.52 & 0.56 & 56.12 \\
\hline & CSF & $0.01 \pm 0.00$ & 0.01 & 0.00 & 0.07 \\
\hline \multirow[t]{2}{*}{ P01M0 } & Plasma & $8.18 \pm 10.45$ & 4.77 & 1.24 & 53.34 \\
\hline & CSF & $0.03 \pm 0.04$ & 0.01 & 0.00 & 0.17 \\
\hline \multicolumn{6}{|c|}{ CHITINASE 3-LIKE PROTEIN 1 ( $\underline{\mathrm{ng}} / \mathrm{ml})}$. \\
\hline \multirow[t]{2}{*}{ Baseline } & Plasma & $96.76 \pm 107.44$ & 60.11 & 23.01 & 573.21 \\
\hline & CSF & $280.08 \pm 62.46$ & 286.47 & 131.11 & 378.19 \\
\hline \multirow[t]{2}{*}{ P01M0 } & Plasma & $135.68 \pm 122.95$ & 96.06 & 30.14 & 524.60 \\
\hline & CSF & $279.96 \pm 61.30$ & 292.23 & 142.02 & 396.58 \\
\hline
\end{tabular}

Abbreviations: $\mathrm{CSF}=$ cerebrospinal fluid, $\mathrm{PO} 1 \mathrm{MO}=$ postoperative 1 month, $\mathrm{SD}=$ standard deviation Table 3 Spearman correlation coefficients of biofluids measured at baseline $(n=29)$ 


\begin{tabular}{ccccccc} 
& $\begin{array}{c}\text { IL-6 } \\
\text { plasma }\end{array}$ & $\begin{array}{c}\text { CRP } \\
\text { plasma }\end{array}$ & $\begin{array}{c}\text { YKL-40 } \\
\text { plasma }\end{array}$ & $\begin{array}{c}\text { IL-6 } \\
\text { CSF }\end{array}$ & $\begin{array}{c}\text { CRP } \\
\text { CSF }\end{array}$ & $\begin{array}{c}\text { YKL40 } \\
\text { CSF }\end{array}$ \\
\hline IL-6 plasma & 1 & $0.55^{*}$ & 0.31 & 0.31 & $0.58^{\star \star}$ & -0.07 \\
CRP plasma & & 1 & $0.45^{\star}$ & 0.17 & $0.70^{\star \star}$ & 0.01 \\
YKL-40 plasma & & & 1 & 0.28 & $0.43^{\star}$ & 0.09 \\
IL-6 CSF & & & & 1 & 0.14 & -0.10 \\
CRP CSF & & & & & 1 & -0.02 \\
YKL-40 CSF & & & & & & 1 \\
\hline
\end{tabular}

${ }^{*} \mathrm{p}<.05, * * \mathrm{p}<.01$

Abbreviations: $C R P=C$-reactive protein, $C S F=$ cerebrospinal fluid, IL-6=interleukin-6, YKL-40= chitinase 3like protein 1 (CHI3L1/YKL-40)

Blue indicates significant ( $\mathrm{p}<.05$ ) within biofluid correlation (i.e., plasma-plasma or CSF-CSF), and red indicates significant between biofluid correlation (i.e., plasma-CSF)

Table 4. Spearman correlation coefficients of biofluids measured on postoperative 1 month $(n=29)$

\begin{tabular}{ccccccc} 
& $\begin{array}{c}\text { IL-6 } \\
\text { plasma }\end{array}$ & $\begin{array}{c}\text { CRP } \\
\text { plasma }\end{array}$ & $\begin{array}{c}\text { YKL-40 } \\
\text { plasma }\end{array}$ & $\begin{array}{c}\text { IL-6 } \\
\text { CSF }\end{array}$ & $\begin{array}{c}\text { CRP } \\
\text { CSF }\end{array}$ & $\begin{array}{c}\text { YKL40 } \\
\text { CSF }\end{array}$ \\
\hline IL-6 plasma & 1 & $0.42^{*}$ & 0.31 & $0.48^{\star \star}$ & $0.47^{\star}$ & 0.29 \\
CRP plasma & & 1 & $0.69^{\star \star}$ & 0.28 & $0.89^{\star \star}$ & 0.22 \\
YKL-40 plasma & & & 1 & 0.26 & $0.59^{\star \star}$ & 0.14 \\
IL-6 CSF & & & & 1 & $0.45^{\star}$ & -0.09 \\
CRP CSF & & & & & 1 & 0.22 \\
YKL-40 CSF & & & & & & 1 \\
\hline
\end{tabular}

${ }^{*} \mathrm{p}<.05, * * \mathrm{p}<.01$

Abbreviations: CRP=C-reactive protein, CSF=cerebrospinal fluid, IL-6=interleukin-6, YKL-40= chitinase 3like protein 1 (CHI3L1/YKL-40)

Blue indicates significant ( $\mathrm{p}<.05$ ) within biofluid correlation (i.e., plasma-plasma or CSF-CSF), and red indicates significant between biofluid correlation (i.e., plasma-CSF)

\section{Figures}




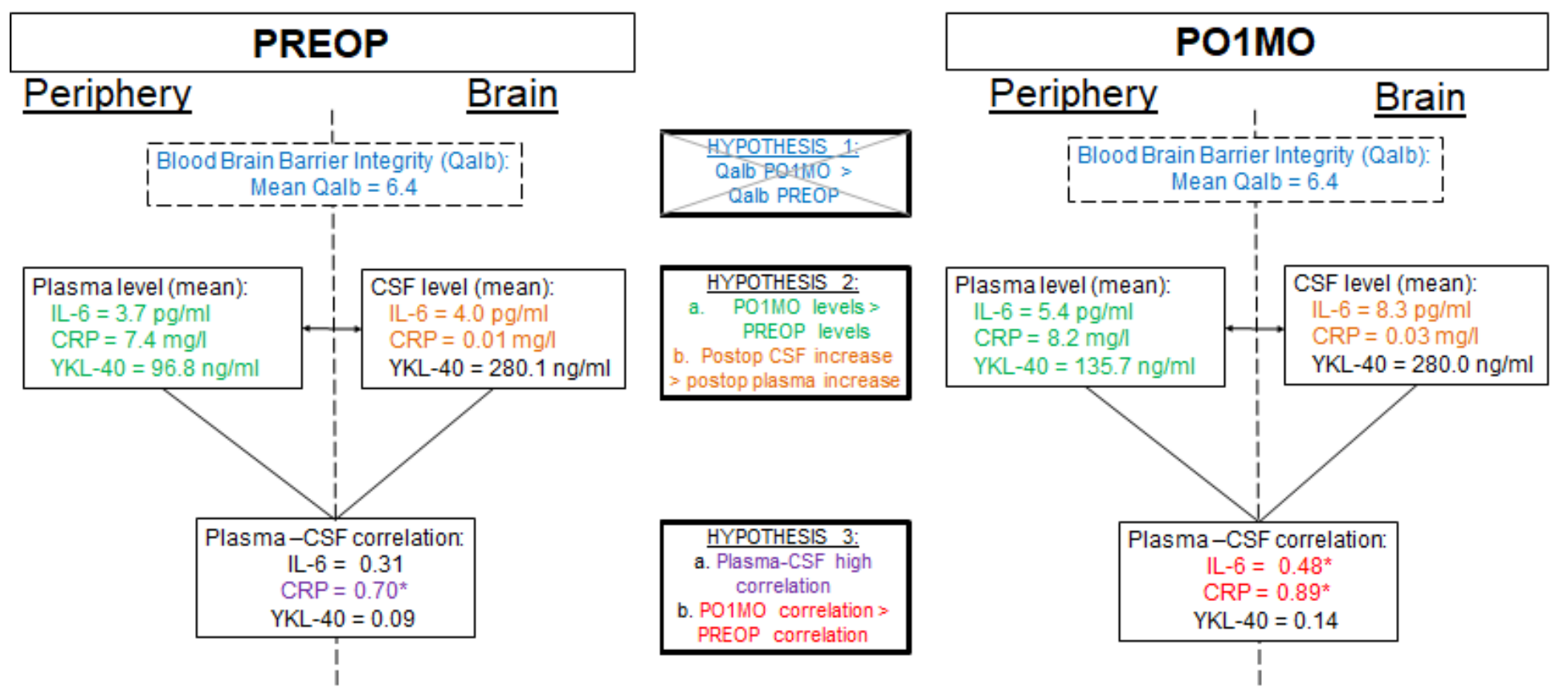

\section{Figure 1}

Summary of findings related to the posited hypotheses. Abbreviations: CRP=C-reactive protein, CSF, IL-6, Qalb, PREOP, P01MO, YKL-40 * $\mathrm{p}<.01$ Each hypothesis and its associated empirical result are show in: blue for Hypothesis 1, green for Hypothesis 2a, orange for Hypothesis 2b, purple for Hypothesis $3 a$, and red for Hypothesis $3 b$.

\section{Supplementary Files}

This is a list of supplementary files associated with this preprint. Click to download.

- Supplmentry.docx 